\title{
VLBI Observations of the Seyfert Galaxy IC 5063
}

T. Oosterloo ${ }^{1}$, A. Tzioumis ${ }^{1}$, J. Reynolds ${ }^{1}$, R. Morganti ${ }^{1,2}$, Z. Tsvetanov ${ }^{3}$, P. $\mathrm{McCulloch}^{4}, \&$ E. King ${ }^{1}$

Abstract. VLBI observations of IC 5063 are presented.

\section{Introduction}

The narrow-line regions (NLR) in Seyfert galaxies are regions of highly ionized, kinematically complicated gas emission. They occupy the central area (to $\sim 1$ $\mathrm{kpc}$ from the nucleus) in the host galaxy and often show a close morphological association with the radio plasma. They represent some of the best examples of regions where interaction between the local ISM and the radio plasma takes place and where shocks appear to be very important. In this respect, obtaining the parameters of the neutral gas is of particular interest. High resolution $\mathrm{H} \mathrm{I}$ observations give the distribution and kinematics of the cold component of the circumnuclear ISM and, therefore, complement the optical data. This has been done for a few objects: in NGC 4151 and in NGC 5252, Pedlar et al. (1992) and Prieto \& Freudling (1996) found a close agreement between the kinematics of the ionized gas and of the neutral hydrogen, strongly supporting the idea that the ionized gas is simply a component of the gaseous disk, ionized by an energy source in the nucleus.

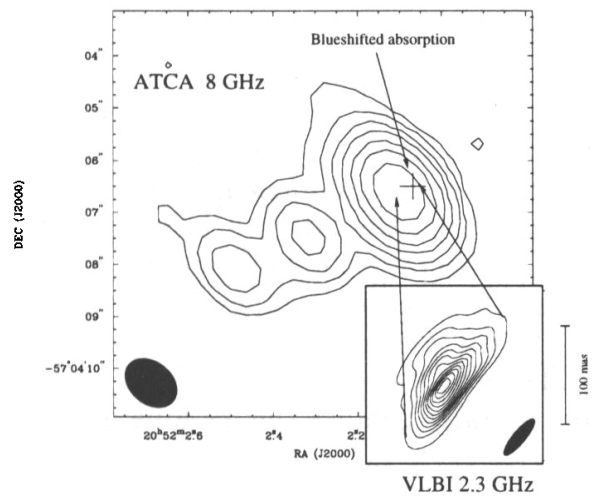

Fig.1

Additionally, $\mathrm{H}$ I line-absorption observations have the advantage of being able to clearly distinguish between infall and outflow of gas along the line of sight to the radio continuum. H I absorption has been detected in a number of Seyfert galaxies (e.g., Gallimore et al. 1994; Brinks \& Mundell 1996), but very broad (i.e. $>$ few hundred $\mathrm{km} \mathrm{s}^{-1}$ ) $\mathrm{H}$ I absorption is observed in a very small subset of Seyfert galaxies. Recently, we have carried out a detailed study of the $\mathrm{HI}$ and the $8-\mathrm{GHz}$ radio continuum in the central region of the nearby $(z=0.0110)$ Seyfert 2 galaxy IC 5063 using the Australia Telescope Compact

\footnotetext{
${ }^{1}$ ATNF-CSIRO, Epping, Australia ${ }^{2}$ Istituto di Radioastronomia, Bologna, Italy ${ }^{3}$ Johns Hopkins University, Baltimore, USA ${ }^{4}$ University of Tasmania, Hobart, Australia
} 
Array (ATCA; Morganti, Oosterloo, \& Tsvetanov 1996, 1997). This is an earlytype galaxy containing a Seyfert source that is particularly strong in the radio continuum $\left(P_{1.4 \mathrm{GHz}}=6.3 \times 10^{23} \mathrm{~W} \mathrm{~Hz}^{-1}\right)$. On the arcsec scale, we find a linear triple structure (Fig. 1) that shows a close spatial correlation with the ionized gas. We identify the nucleus to be the central blob. Coincident with the western "lobe" we find very broad ( $\left.700 \mathrm{~km} \mathrm{~s}^{-1}\right)$, mainly blueshifted $\mathrm{H} \mathrm{I}$ absorption. The large width is unlikely to be due to gravitational motion. Thus, in IC 5063 we see clear evidence, both morphological and kinematical, for strong shocks resulting from the interaction between the radio plasma and the interstellar medium in the central few kiloparsecs. In the optical, blue-shifted emission lines with similar width are observed at the same position (Wagner \& Appenzeller 1989), consistent with the idea that the absorption is against the western blob. However, we estimate the energy flux in the radio plasma to be an order of magnitude smaller than the energy flux emitted in emission lines. The shocks associated with the jet-ISM interaction are, therefore, unlikely to account for the ionization of the NLR (unless the lobe plasma contains a significant thermal component; Bicknell et al., in prep.) and the NLR must be photoionized by the nucleus.

\section{The Sub-kpc Structure: VLBI Observations}

The above observations are still limited by low resolution and therefore VLBI observations were carried out. IC 5063 was observed with the Australian LBA at $2.3 \mathrm{GHz}$ using the $\mathrm{S} 2$ recording system. The final map, shown in the insert of Fig. 1, has a beam of $\sim 56 \times 15$ mas. From registering the two images, we conclude that the VLBI source coincides with the brightest blob in the $8-\mathrm{GHz}$ ATCA map. The spectrum and the brightness temperature of the VLBI source also suggest that it is unlikely to be the nucleus. The spectral index of inferred from the ATCA 8.6 and $1.4-\mathrm{GHz}$ images $(\alpha \sim-1)$, is more consistent with a compact radio lobe or jet than an $A G N$ nucleus, which generally exhibit flat or inverted radio spectra. Similarly, the inferred brightness temperature of the VLBI source of $T_{B} \sim 10^{7} \mathrm{~K}$ is several orders of magnitude less than the typical values seen in $\sim$ milliarcsecond $A G N$ cores or inner (pc-scale) jets $\left(10^{9} \sim 10^{11} \mathrm{~K}\right.$ ). Note also the difference in the p.a. of the arcsec structure compared to the VLBI structure (p.a. $\sim 160^{\circ}$ ). The next step will be to do H I VLBI observations to check if the $\mathrm{H}$ I absorption is really against the $\mathrm{W}$ lobe as described above.

\section{References}

Brinks, E., \& Mundell, C. G. 1996. In The Minnesota Lectures on Extragalactic Neutral Hydrogen, ed. E. D. Skillman, ASP Conf. Series, 106, 268-292.

Gallimore, J. F., et al. 1994. ApJ, 422, L13-16.

Morganti, R., Oosterloo, T., \& Tsvetanov, Z. 1996. In "Emission lines in Active Galaxies: New Methods and Techniques", eds. B. M. Peterson, F.-Z. Cheng, \& A. S. Wilson, ASP Conf. Series, 113, 310-311.

Morganti, R., Oosterloo, T., \& Zsvetanov, Z. 1997. AJ, accepted.

Pedlar, A., et al. 1992. MNRAS, 259, 369-380.

Prieto, M. A., \& Freudling, W. 1996. MNRAS, 279, 63-71.

Wagner, S.J., \& Appenzeller, I. 1989. A $8 A$, 225, L13-16. 